Gut, 1980, 21, 475-479

\title{
Effects of wide variations in portal pressure on mesenteric blood flow and absorption from the canine colon
}

\author{
A S GRANDISON, I D HARRISON, AND R SHIELDS \\ From the Department of Surgery, University of Liverpool, Liverpool
}

SUMMARY Splanchnic blood flow was studied over a wide range of portal pressures in five dogs. An inverse linear relationship between flow and pressure was found in the range from 6 to $42 \mathrm{mmHg}$. The colonic absorption of ammonia, water, sodium, potassium, bicarbonate, and chloride was measured in four dogs over a similar range of portal pressures. Absorption of ammonia, water, sodium, and chloride was significantly reduced by increasing portal pressure, and this reduction was abolished when portal pressure was allowed to return to the basal level. It is suggested that splanchnic decompression by portasystemic shunt gives rise to increased mesenteric flow and increased colonic ammonia absorption, which may contribute to portasystemic encephalopathy.

After portasystemic shunting in the treatment of portal hypertension, the concentration of ammonia in the blood is markedly increased. ${ }^{12}$ There may be several explanations for this observation. One possibility is that, with splanchnic decompression, ammonia is more rapidly absorbed from the intestine in which it is produced. Previous work in this laboratory-indirect studies in $\operatorname{man}^{2}$ and direct ones in the dog $^{3}$-suggests that this may be so. However, in the studies in dogs the portal pressure was reduced from normal to subnormal values and clearly this is not relevant to patients with oesophageal varices where pathologically high levels of portal pressure are reduced to normal.

In this study the absorption of ammonia and other substances from the canine colon was measured over a broad range of portal pressure. At the same time the effects of these variations in portal pressure on splanchnic blood flow were studied.

\section{Methods}

\section{ANIMALS}

Labrador dogs weighing $15-20 \mathrm{~kg}$ were fasted for 20 hours before the experiments, but water was not withheld. Anaesthesia was induced with sodium thiopentone and maintained with halothane, nitrous oxide, and oxygen throughout all surgical procedures

Received for publication 5 November 1979 and measurement of colonic absorption. The dogs were killed immediately after completion of the experiments.

\section{SURGICAL PROCEDURES}

With the animal lying supine, the abdomen was opened through a mid-line incision. A Dunn vessel occluder (J G Franklin, High Wycombe, Bucks) was placed around a segment of the portal vein immediately caudal to the liver. The occluder is a circular pneumatic cuff which can be inflated or deflated to produce controlled changes in portal pressure.

A mesenteric branch of the portal vein and a femoral artery were cannulated to permit measurement of portal pressure and systemic arterial pressure.

A $4 \mathrm{~mm}$ probe was fitted around the superior mesenteric artery for the measurement of blood flow using a Biotronex BL 613 sine-wave flowmeter.

An intestinal cannula was inserted into the proximal colon and the bowel tied to lucite bobbins carried on the shaft of the cannula (Fig. 1) so that solutions could be introduced and withdrawn from the lumen without leakage.

The abdomen was then closed and after one hour experiments were begun.

EXPERIMENTAL PROTOCOL

Simultaneous measurements of portal pressure and flow in the superior mesenteric artery were made, 


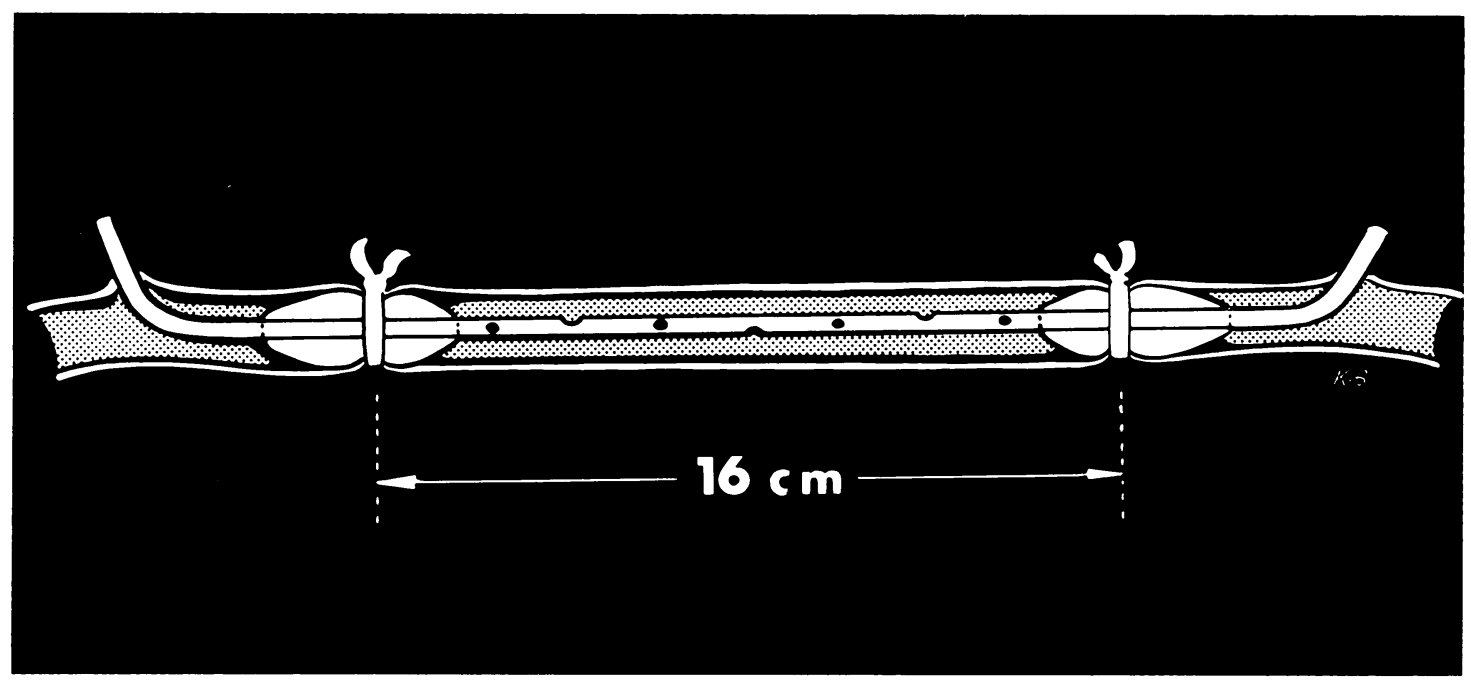

Fig. 1 The position of the intestinal cannula in the colon segment during absorption studies.

allowing a five minute period for stabilisation between inflation or deflation of the vessel occluder and measurement of pressure. Adjustments were made in a randomised manner.

Measurements of the rates of absorption of the various substances were carried out in duplicate at various portal pressures - basal with the cuff uninflated or increased with the portal vein partially occluded by the inflated cuff. The order of the experiments-whether portal pressure was basal or increased--was selected randomly.

After the absorption tests were completed, the segment of colon was excised and opened along its antimesenteric border. The dimensions were measured so that the rate of absorption could be related to the surface area.

\section{ABSORPTION MEASUREMENTS}

All solutions to be introduced into the colon were maintained at $37 \mathrm{C}$. Before the absorption tests were started, the colon segment was thoroughly rinsed with 11 saline solution $(0.9 \% \mathrm{w} / \mathrm{v})$ followed by $100 \mathrm{ml}$ test solution $\left(25 \mathrm{mmol} / 1 \mathrm{NH}_{4} \mathrm{Cl} ; 3.5 \mathrm{mmol} / 1\right.$ $\mathrm{KCl} ; 117 \mathrm{mmol} / \mathrm{l} \mathrm{NaCl}$ adjusted to $\mathrm{pH} 7.0$ with $0.1 \mathrm{mmol} / 1 \mathrm{NaOH})$. This particular concentration of ammonia was chosen because it lies at the upper limit of the faecal concentration in normal subjects and in the lower range of the concentration observed in cirrhotic patients, as determined by in vivo dialysis. ${ }^{4}$

The segment was then emptied as completely as possible and $22 \mathrm{ml}$ test solution containing $0.25 \mu \mathrm{Ci}$ ${ }^{51} \mathrm{Cr}$-EDTA as a non-absorbable volume marker was introduced and thoroughly mixed by repeated withdrawal and reinstillation for 30 seconds. A $2 \mathrm{ml}$ aliquot was removed for radioactive counting and measurement of $\mathrm{pH}$. The contents of the segment were mixed at 10 minutes and withdrawn at the end of 20 minutes.

The aspirates were centrifuged before chemical analysis, radioactive counting, and measurement of $\mathrm{pH}$. The segment was rinsed with $100 \mathrm{ml}$ of unlabelled test solution before the next absorption test.

\section{ANALYTICAL METHODS}

The ammonia concentrations of the aspirates and test solutions were estimated by the method of Fawcett and Scott. ${ }^{6}$ The sample was mixed with a solution containing phenol, sodium nitroprusside, sodium hypochlorite, and sodium hydroxide and allowed to stand for 30 minutes at $37^{\circ} \mathrm{C}$. The intensity of colour of the resulting blue solution was measured against a reagent blank using a Unicam SP600 spectrophotometer at $624 \mathrm{~nm}$. Concentrations of sodium and potassium were measured by means of a flame photometer and concentrations of chloride were determined with a chloride meter. The concentrations of bicarbonate were measured by the method of Van Slyke (Harleco $\mathrm{CO}_{2}$ apparatus). The radioactivity of the solutions was determined in a Nuclear Chicago model 4233 gamma counter.

CALCULATION OF RESULTS AND

STATISTICAL ANALYSIS

The rate of water absorption was calculated from changes in the concentrations of the non-absorbed 
volume marker $\left({ }^{51} \mathrm{Cr}-\mathrm{EDTA}\right)$ using the equation:

$V_{\text {net }}=\frac{\left[\left(\frac{22 R_{I}}{R_{D}}-2\right)-\left(\frac{22 R_{I}-2 R_{D}}{R_{T}}\right)\right]}{S}$

Where $\mathrm{V}_{\mathrm{n} e t}$ is the net absorption of water in $\mathrm{ml} / \mathrm{cm}^{2} / 20 \mathrm{~min}$

$\mathrm{S}$ is the surface area of the loop $\left(\mathrm{cm}^{2}\right)$

$R_{I}$ is the radioactive concentration of the test solution $(\mathrm{cpm} / \mathrm{ml})$

$R_{D}$ is the radioactive concentration of the loop contents after the initial mixing (cpm/ml)

$R_{T}$ is the radioactive concentration of the final aspirate $(\mathrm{cpm} / \mathrm{ml})$.

The rate of net absorption of ammonia and other electrolytes was calculated from the following equation:

$$
A=\frac{\left[C_{0}\left(\frac{22 R_{I}}{R_{D}}-2\right)-C_{T}\left(\frac{22 R_{I}-2 R_{D}}{R_{T}}\right)\right]}{S}
$$

Where $\mathrm{A}$ is the net absorption in $\mu \mathrm{mol} / \mathrm{cm}^{2} / 20 \mathrm{~min}$ $\mathrm{C}_{\mathrm{o}}$ is the concentration of the appropriate substance in the test solution $(\mathrm{mmol} / \mathrm{l})$

$\mathrm{C}_{\mathbf{T}}$ is the concentration in the final aspirate $(\mathrm{mmol} / \mathrm{l})$

The results of the haemodynamic studies and the changes in absorption in relation to changes in portal pressure were subjected to regression analysis and analysis of covariance as described by Snedecor and Cochran.?

\section{Results}

\section{HAEMODYNAMIC STUDIES}

A clear inverse linear relationship between pressure and flow was observed in each dog in the range from 6 to $42 \mathrm{mmHg}$ (the results from five dogs are shown in Fig. 2).

By applying regression analysis to the data this relationship between pressure and flow in each of the five dogs was found to be significant $(P<0.01$ in all cases). Significant variation of the relationship between individual animals was demonstrated by analysis of covariance $(\mathrm{F}=27.59, \mathrm{P}<0.001)$.

In addition, it was shown that these changes in flow were fully reversible, as flow returned to its basal level within five minutes of deflation of the occluder.

\section{ABSORPTION STUDIES}

At basal portal pressures in the four dogs, water, sodium, chloride, and ammonia were absorbed,

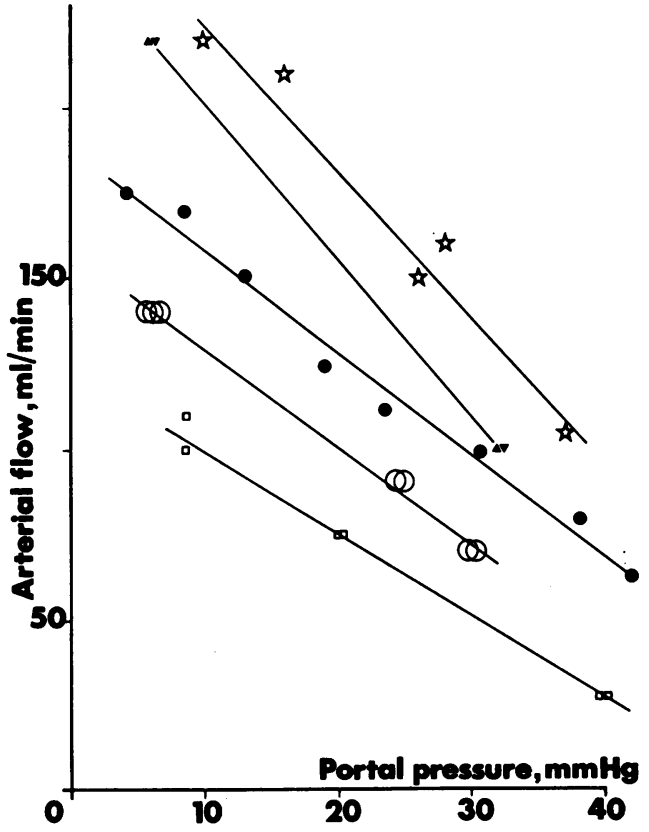

Fig. 2 The relationship between portal pressure and flow in the superior mesenteric artery in five dogs.

whereas potassium and bicarbonate were secreted by the colon (Table).

The rates of absorption of water, ammonia, sodium, and chloride were significantly reduced at raised portal pressure (Figs. 3-6) as determined by analysis of covariance. The rates of secretion of potassium and bicarbonate, however, were not significantly affected.

For ammonia, the change in the rate of absorption was plotted against increase in portal pressure. The regression line was calculated to have a gradient of $0.53 \mu \mathrm{mol} / \mathrm{cm}^{2} / 20 \mathrm{~min} / \mathrm{mmHg}$. Therefore, given a mean rate of ammonia absorption of $2.52 \mu \mathrm{mol} / \mathrm{cm}^{2} /$ $20 \mathrm{~min}$ at basal portal pressure, increases in portal pressure of 10,20 , and $30 \mathrm{mmHg}$ would be associated

Table Colonic absorption or secretion of water and electrolytes at basal portal pressures in four dogs

\begin{tabular}{ll}
\hline Substance & $\begin{array}{l}\text { Absorption } / \mathrm{cm}^{2} / 20 \mathrm{~min} \\
(\mu \mathrm{mol})\end{array}$ \\
\hline Water & $+0.037 \pm 0.0037 \mathrm{ml}$ \\
Ammonia & $+2 \cdot 52 \pm 0.18$ \\
Sodium & $+2 \cdot 25 \pm 0.57$ \\
Potassium & $-0.22 \pm 0.10$ \\
Bicarbonate & $-1.79 \pm 0.20$ \\
Chloride & $+7.27 \pm 0.55$ \\
\hline
\end{tabular}

+ ve sign denotes absorption.

-ve sign denotes secretion.

All values mean \pm SEM. 


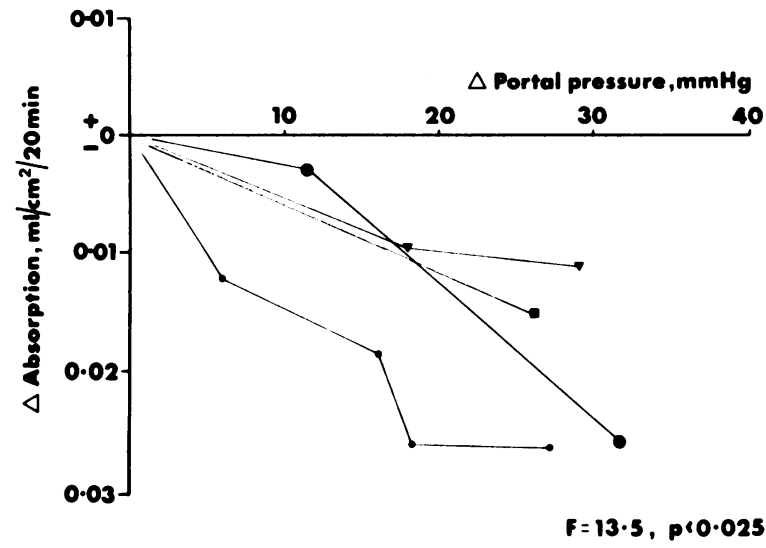

Fig. 3 The relationship between changes in water absorption and changes in portal pressure in four dogs.

with 20,40 , and $60 \%$ decreases in the rate of ammonia absorption.

$\mathrm{pH}$ was not significantly altered during the absorption tests.

\section{Discussion}

The first striking finding in this study is that, by increasing the pressure in the portal vein, mesenteric blood flow is reduced. This relationship has been demonstrated between portal pressures of 6-42 $\mathrm{mmHg}$ and is reversible. Previously, it has been assumed that increased portal pressure results in a reduction in mesenteric blood flow. ${ }^{8}$ Moreover, it has been demonstrated that the relief of portal hypertension by portasystemic shunting results in a large increase in mesenteric blood flow. ${ }^{910}$ To our knowledge, however, the relationship between portal pressure and mesenteric blood flow has not previously been fully defined.

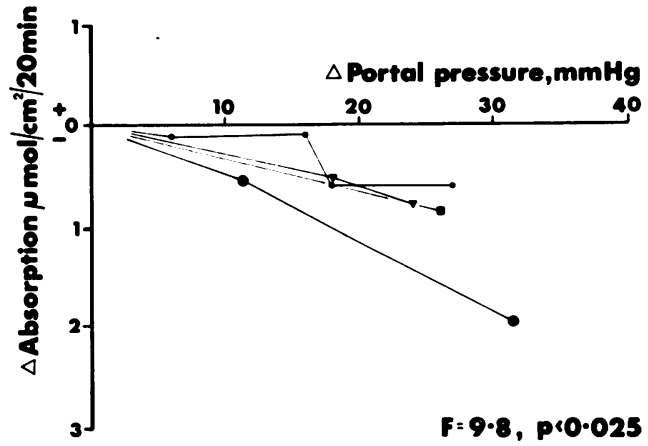

Fig. 4 The relationship between changes in ammonia absorption and changes in portal pressure in four dogs.

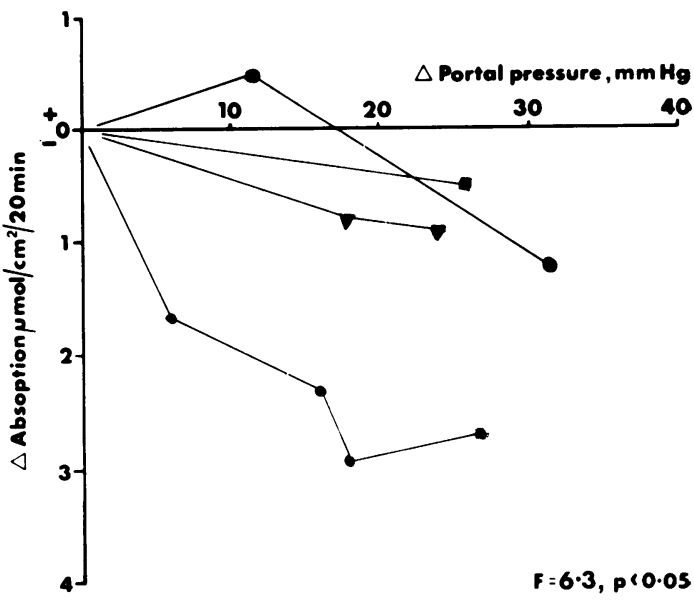

Fig. 5 The relationship between changes in sodium absorption and changes in portal pressure in four dogs.

However, it must be emphasised that the present studies are acute experiments in dogs and the results cannot be extrapolated to cirrhotic patients with portal hypertension where compensatory mechanisms, such as the formation of collateral channels, may minimise this decrease in splanchnic flow. However, the haemodynamic consequences of a portacaval shunt are sudden and from these data it would be expected that a rapid fall in portal pressure of $30 \mathrm{mmHg}$ after operation would be associated with a large increase in mesenteric flow, possibly to more than double the pre-shunt value. This suggestion conforms to the clinical findings that

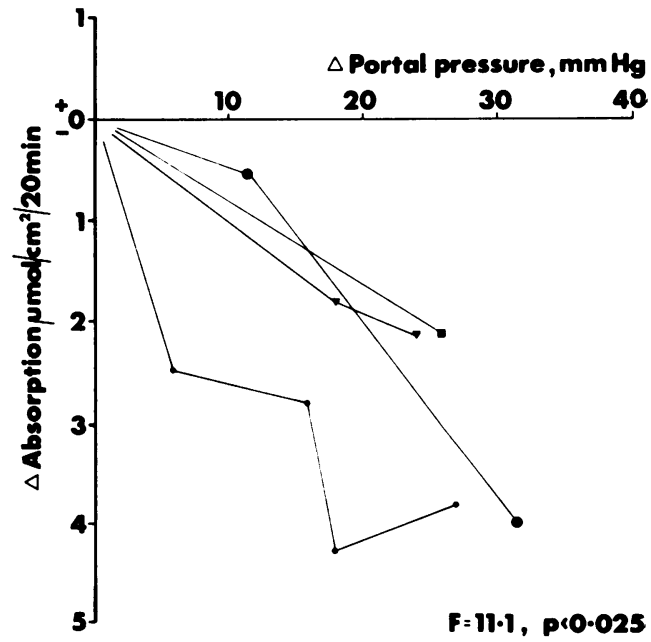

Fig. 6 The relationship between changes in chloride absorption and changes in portal pressure in four dogs. 
portasystemic shunting results in an approximate doubling of total portal venous flow. ${ }^{9}{ }^{10}$ The present studies cannot determine whether the mucosal microcirculation in the colon is similarly affected by changes in portal pressure, as would seem likely, but work is currently in progress to establish this.

To our knowledge, the effect of raising portal pressure on colonic absorption of ammonia has not previously been investigated. The jejunal absorption of ammonia was said to be enhanced by portal hypertension and that this enhancement was abolished by portacaval shunt. ${ }^{11}$ On the other hand, Enquist et al. ${ }^{8}$ reported that xylose absorption from the ileum was diminished by raising the portal pressure and we have previously demonstrated that ammonia absorption from the colon was enhanced after portacaval shunt. ${ }^{3}$

Shields and Code $\mathrm{C}^{12}$ previously showed that absorption of sodium and water from the small intestine was reduced by raising portal pressure by a vessel occluder. The results of the present studies have extended these observations by showing that the rates of colonic absorption of ammonia, water, sodium, and chloride are all reduced at raised portal pressure, and return to normal when portal pressure returns to the basal level.

Of particular note is that the effect of increased portal pressure affects not only sodium, which is actively and rapidly absorbed by the intestine, but also ammonia whose transport is slower, passive, and presumably unidirectional.

Absorption of ammonia may be reduced by up to $60 \%$ when portal pressure is increased over a short period of time by about $30 \mathrm{mmHg}$. When portal pressure is reduced by $20-30 \mathrm{Hg}$, as often occurs during portasystemic shunting, absorption of ammonia would be expected from these results to be grossly enhanced possibly to more than double the pre-shunt level. This increased absorption of ammonia would contribute to the raised concentrations of ammonia in the systemic circulation and may be of significance to the causation of hepatic encephalopathy. However, caution must be observed in extending conclusions or speculation from acute studies in the dog such as these, to the chronic situation in the cirrhotic patient with portal hypertension.

The authors wish to thank Miss A Birch and Mr J Yates for their expert technical assistance. The research was supported by a grant from the Medical Research Council.

\section{References}

${ }^{1}$ Walker CO, Schenker S. Pathogenesis of hepatic encephalopathy with special reference to the role of ammonia. Am J Clin Nutr 1970; 23: 619-32.

${ }^{2}$ Sein MM, Hardy-Smith A, Shields R. The effect of portacaval shunt on colon transfer of ammonia in cirrhosis of the liver (abstract). Gut 1974; 15 : 837.

${ }^{3}$ Harrison ID, Hardy-Smith A, Shields R. Ammonia absorption from the canine colon after portacaval shunt. Br J Surg 1977; 64: 851-6.

${ }^{4}$ Sein MM. The effect of portal systemic shunt on colon transfer of ammonia in cirrhosis of the liver. Ch.M. thesis, Liverpool University 1974.

${ }^{5}$ Brigham KL, Banwell JG, Pierce NF, Mitra RC, Fedson DS, Mondal A. Indicator dilution studies in the small bowel of patients with cholera diarrhea. Johns Hopkins Med J 1970; 127: 97-118.

${ }^{6}$ Fawcett JK, Scott JE. A rapid and precise method for the determination of urea. J Clin Pathol 1960; 13 156-9.

${ }^{7}$ Snedecor GW, Cochran WG. Statistical methods. 6th Ed. Ames, Iowa: Iowa State University Press, 1967. ${ }^{8}$ Enquist IF, Golding MR, Aiello RG, Fierst SM, Solomon NA. The effect of portal hypertension on intestinal absorption. Surg Gynecol Obstet 1965; 120: 87-91.

${ }^{9}$ Ferguson DJ. Hemodynamics in surgery for portal hypertension. Ann Surg 1963; 158: 383-6.

${ }^{10}$ Price JB Jr, McCullough W, Peterson L, Britton RC, Voorhees AB Jr. Effects of portal systemic shunting on intestinal absorption in the dog and in man. Surg Gynecol Obstet 1967; 125: 305-10.

${ }^{11 W i n d s o r ~ C W, ~ G o o d h e a d ~ B, ~ O r l o f f ~ M J . ~ E f f e c t s ~ o f ~}$ portacaval shunts and experimental liver disease on intestinal absorption of ammonia. Surgical Forum 1968; 19: 333-5.

${ }^{12}$ Shields R, Code CF. Effects of increased portal pressure on sorption of water and sodium from the ileum of dogs. Am J Physiol 1961 ; 200 : 775-80. 